\title{
Synchronization induced by signal propagation delays in inhibitory networks
}

\author{
Shivakeshavan Ratnadurai ${ }^{2}$, Sachin S Talathi ${ }^{1 *}$, Paul R Carney ${ }^{1,3}$, Pramod P Khargonekar ${ }^{2}$ \\ From Nineteenth Annual Computational Neuroscience Meeting: CNS*2010 \\ San Antonio, TX, USA. 24-30 July 2010
}

It is generally accepted that inhibitory neurons play an important role in the generation of synchronous brain rhythms. A number of theoretical studies have been conducted over last decade to determine the properties of the inhibitory neuronal networks that can result in stable network synchrony and much work has focused on synaptic properties of GABA inhibition (the decay time of the synapse and the reversal potential), the heterogeneity in the intrinsic neuron firing rates and the architecture of the underlying networks. Very few studies, however, have focused on the role for explicit signal propagation delays in modulating synchrony in inhibitory networks. One reason for the paucity of work in this area stems for the fact that mathematical studies of a general network of coupled nonlinear delay- differential equations resulting from the presence of signal propagation delays is a formidable task. Here our goal is to systematically investigate, in the setting of computer simulations, the role of signal propagation delays in the synchronization of inhibitory networks.

We consider a random network $G$ of $N=10$ interacting inhibitory neurons (Type-1 parvalbumin positive interneurons) with the following network constraints: (1) Unidirectional neuronal interaction (2) Mean in-degree for each node of the network is $k=3$. In order to systematically investigate how neuronal synchrony in this random network changes as a function of signal propagation delay $\tau_{\mathrm{s}}, 1000$ instances of $G$ were generated and each network was simulated 100 times with random initial conditions. All simulations were performed for fixed

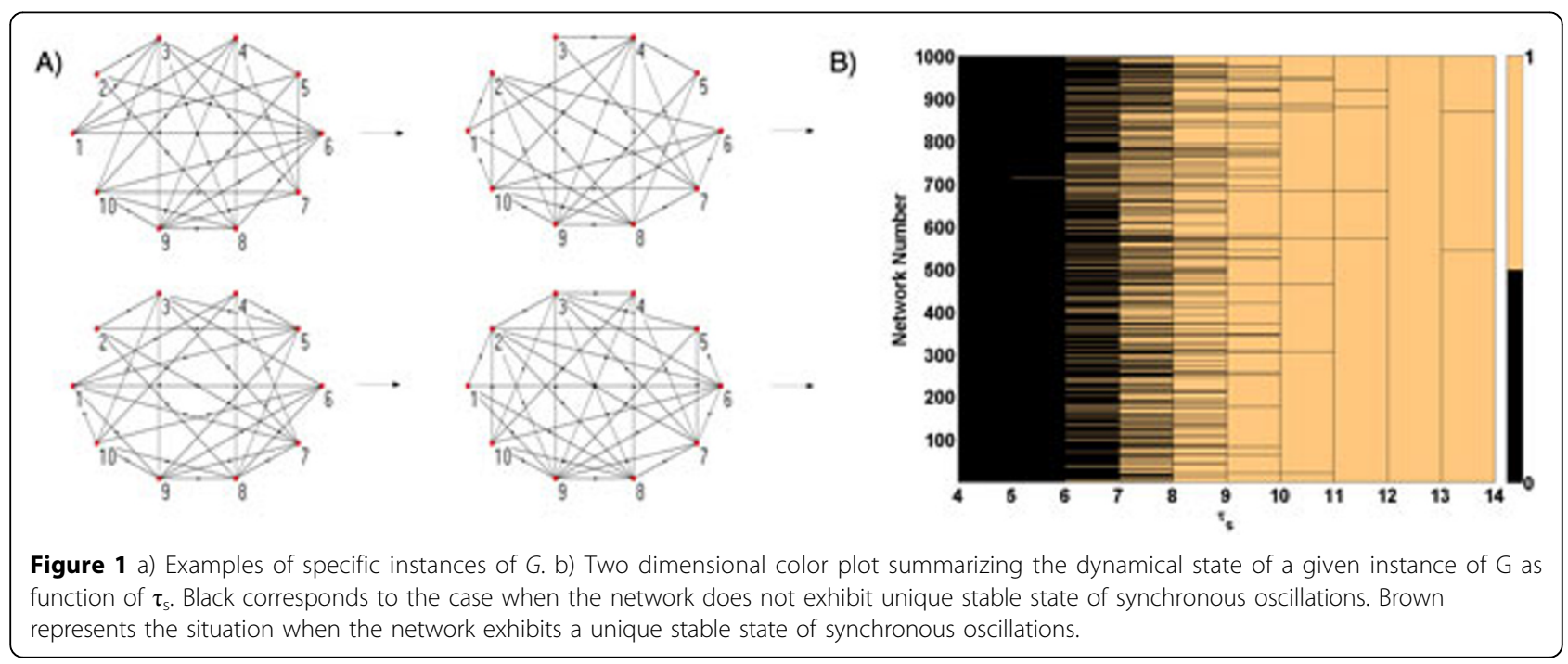

* Correspondence: sachin.talathi@bme.ufl.edu

'Department of Biomedical Engineering, University of Florida, Gainesville, Florida, 32611, USA 
synaptic parameters: the synaptic conductance $g=0.1 \mathrm{mS}$ / $\mathrm{cm}^{2}$, reversal potential $E_{R}=-75 \mathrm{mV}$, synaptic decay time $\tau_{d}=10 \mathrm{~ms}$, and the intrinsic period of oscillation for neuron $T_{O}=33 \mathrm{~ms}^{-1}$.

In Figure 1A, we show examples of instances of $\mathrm{G}$ satisfying the constraints described above. The key result of our simulation experiments is summarized in Figure 1B. For zero propagation delay none of the 1000 instances of $G$ produced complete synchrony. This observation can be explained mathematically by considering the Arnold's tongue for two uni-directionally coupled interneurons [1]. As the signal propagation delay increases, the fraction of networks that completely synchronize independent of the initial conditions increases. For signal propagation delay of $\tau_{s}=12 \mathrm{~ms}$, all the 1000 instances of $\mathrm{G}$ completely synchronize independent of initial conditions. The number of $G$ instances that exhibit complete synchrony drops as $\tau_{s}$ is increased beyond $12 \mathrm{~ms}$. In addition, the period of synchronous oscillation is $40.2 \mathrm{~ms}$ for all 1000 instances of $\mathrm{G}$ at $\tau_{s}=12 \mathrm{~ms}$. This observation suggests that there is a unique value for signal propagation delay for which all random networks subject to the constraints mentioned above exhibit a "single stable state of synchrony." This result is in sharp contrast to multi-stable attractors observed in neuronal networks with large signal propagation delays. This rather striking phenomenon is a peek at the very important role of delays in synchrony in inhibitory neuronal networks.

\section{Acknowledgements}

This work was funded in part by the NIH grants R01-EB004752 and R01EB007082, the Wilder Center of Excellence for Epilepsy Research and Eckis Professor Endowment to PPK.

\section{Author details}

${ }^{1}$ Department of Biomedical Engineering, University of Florida, Gainesville, Florida, 32611, USA. ${ }^{2}$ Department of Electrical and Computer Engineering, University of Florida, Gainesville, 32611, USA. ${ }^{3}$ Department of Pediatrics

Neurology and Neuroscience, University of Florida, Gainesville, 32611,USA

Published: 20 July 2010

\section{Reference}

1. Talathi S S, Khargonekar P: Predicting synchrony in simple inhibitory network, Perspectives in Mathematical System Theory, Control, and Signal Processing. Springer Verlag 2010.

doi:10.1186/1471-2202-11-S1-P2

Cite this article as: Ratnadurai et al:: Synchronization induced by signal propagation delays in inhibitory networks. BMC Neuroscience 2010 11(Suppl 1):P2.

\section{Submit your next manuscript to BioMed Central} and take full advantage of:

- Convenient online submission

- Thorough peer review

- No space constraints or color figure charges

- Immediate publication on acceptance

- Inclusion in PubMed, CAS, Scopus and Google Scholar

- Research which is freely available for redistribution

Submit your manuscript at www.biomedcentral.com/submit 